\title{
The activity of factor VIII and IX of cord blood at mid-trimester in fetuses without hemophilia
}

\author{
David Kwang Yong Choe', Jeong Won $\mathrm{Oh}^{\dagger}$, Jong Kwan Jun*, and Young Min Choi \\ Department of Obstetrics and Gynecology, Seoul National University College of Medicine, Seoul, Korea
}

\begin{abstract}
Purpose: Molecular genetic analysis is the main approach used for prenatal diagnosis of hemophilia A and B. However, in certain cases, such analysis is uninformative. In such situations, direct measurement of fetal coagulation factor levels is still the best option, and it may be the only option in some cases. This study was conducted to determine the normal ranges of midtrimester cord blood factor VIII (FVIII) and IX (FIX) in a Korean population.

Materials and Methods: Twenty-six FVIII samples and 29 FIX samples were assayed in fetal cord blood acquired by ultrasound-guided cordocentesis. Sampling was conducted during gestational ages of 19-24 weeks.

Results: The mean and standard deviations for FVIII and FIX activity were $45.5 \pm 30.5 \%$ and $19.9 \pm 12.2 \%$, respectively. Ranges for FVIII and FIX were $1.5-125.0 \%$ and $6.0-52.0 \%$, respectively.

Conclusion: Our study revealed the normal ranges and lowest level of factor VIII and factor IX in non-affected normal fetus by fetal cord blood sampling during the mid-trimester in a Korea population. The factor assay of the fetal cord blood is invasive but feasible and provides important basic data related to hemophilia.
\end{abstract}

Key words: Hemophilia, Blood coagulation factors, Cordocentesis, Prenatal diagnosis.

\section{Introduction}

Hemophilia is defined as a group of bleeding disorders characterized by deficiencies in coagulation factors. The most common types are hemophilia A and B, which either lack factor VIII (FVIII) or factor IX (FIX), respectively. They are genetic disorders with $\mathrm{X}$-linked recessive inheritance, and their prevalence is about 1 in 10,000 overall and 1 in 5,000 in the male population [1]. In Korea, there were approximately 2,000 patients with hemophilia in 2007, according to the Korean Hemophilia Foundation 2007 annual report [2].

The severity of the disease is correlated with the plasma concentrations of the affected coagulation factors. Severe hemophilia is defined as having less than $1 \%$ of normal plasma concentrations [3]. Patients with plasma concentrations less than $5 \%$ or $7 \%$ but equal to or greater than $1 \%$ of the normal levels are classified as having moderately severe hemophilia; those with higher concentrations are categorized as having mild hemophilia [3]. Among patients with hemophilia A, 35\% have severe, $15 \%$ have moderate, and $55 \%$ have mild forms of the disease [4]. Although patients with mild hemophilia rarely encounter spontaneous bleeding episodes and the condition can sometimes even go unnoticed, severe forms of the disease cause recurrent bleeding in the joints, connective tissues, and other systems. These patients require lifelong treatment, with the main principle behind these therapies being the replacement

\footnotetext{
Received: 26 October 2016, Revised: 23 December 2016, Accepted: 23 December 2016, Published: 31 December 2016

*Corresponding author: Jong Kwan Jun, M.D., Ph.D.

Department of Obstetrics and Gynecology, Seoul National University College of Medicine, 103 Daehak-ro, Jongno-gu, Seoul 03080, Korea.

Tel: +82-2-2072-3744, Fax: +82-2-762-3599, E-mail: jhs0927@snu.ac.kr

tThese authors contributed equally to this work as co-first authors.

Conflict of interest: The authors declare that they do not have any conflicts of interest.
}

(c) This is an open-access article distributed under the terms of the Creative Commons Attribution Non-Commercial License (http://creativecommons.org/licenses/by-nc/4.0/) which permits unrestricted non-commercial use, distribution, and reproduction in any medium, provided the original work is properly cited.

(c) Copyright 2016 by the Korean Society of Medical Genetics 
of deficient coagulation factors.

Considering the difficulties experienced by patients with hemophilia and their families, prenatal diagnosis of this disease plays a crucial role in the early diagnosis of the disease. Clotting factor levels in fetal blood samples can be directly measured using cordocentesis. This direct measurement approach to examine fetal clotting factors was employed in the prenatal diagnosis of hemophilia well before the use of molecular genetic diagnosis $[5,6]$. Although it is an invasive method, it remains a viable option.

There have been numerous efforts to determine the normal ranges of fetal FVIII and FIX activity levels in several countries outside of Korea [5-7]. Here, for the first time, we evaluated fetal coagulation factor levels mid-trimester to determine the normal ranges that can be used in the prenatal diagnosis of hemophilia.

\section{Materials and Methods}

\section{Study design}

We enrolled singleton pregnancies that went through the cordocentesis procedure for prenatal diagnosis of hemophilia between 1996 and 1999 at the Seoul National University Hospital (Seoul, Korea). The indicators for fetal blood sampling were cases of pregnant carriers of hemophilia A or B, results of mosaicism in the analysis of amniotic fluid for karyotyping, confirmation of rubella infection, and cases of major fetal anomaly for assessing the risk of chromosomal abnormality between the gestational ages of 19 and 24 weeks. We excluded cases in which the affected hemophilia status was confirmed after delivery. In all of these cases, unaffected hemophilia status was confirmed by clotting factor assays after delivery. The Institutional Review Board of the Seoul National University Hospital accepted this study: institutional review board No. 1406084589.

\section{Fetal blood sampling and clotting factor assays}

Fetal blood sampling was done by direct puncture of the umbilical vein using ultrasound-guided cordocentesis. To eliminate the possibility of maternal contamination, most samplings were done in floating umbilical cord and confirmed by a mean corpuscular volume (MCV) of red blood cells greater than $110 \mu \mathrm{m}^{3}$. Nine volumes of cord blood were collected in 1 volume of $0.109 \mathrm{M} \mathrm{(3.2 \% )} \mathrm{trisodium} \mathrm{citrate} \mathrm{anticoagulant} \mathrm{in} \mathrm{a}$ 2.7- $\mathrm{mL}$ vacutainer. The samples were centrifuged at $2,500 \mathrm{~g}$ for 10 minutes at room temperature $\left(18-25^{\circ} \mathrm{C}\right)$ and the coagulant activities of FVIII and FIX were measured by the one-stage assay method [8] with a coagulometer using commercial kits (Diagnostica Stago, Asnière, France) [9].

\section{Statistics}

The distributions of the variables were tested for deviations from the normal range by using the Shapiro-Wilk statistic. Mann-Whitney $U$ tests were used to compare the difference of the median levels of FVIII and FIX in accordance of gestational period. Spearman correlation was employed to examine the correlation among the levels of FVIII and FIX and the gestational age (IBM SPSS Statistics ver. 20.0; IBM Co., Armonk, NY, USA). Significance was assumed for a $P$-value of $<0.05$.

\section{Results}

A total of 30 women underwent the cordocentesis procedure for antenatal diagnosis of various disorders. Two fetuses that were found to be affected by severe hemophilia A after delivery were excluded; there were no cases found to be affected by severe hemophilia B. Out of the unaffected fetuses, 26 cases were evaluated for coagulation FVIII, and 29 cases were evaluated for coagulation FIX. Table 1 shows the characteristics and normal range of fetal coagulation FVIII and FIX between the gestational ages of 19 to 24 weeks. The mean age was 21.4 weeks of gestation at the time of the cordocentesis procedure. All of these samples were confirmed to not have maternal contamination by examining the MCV levels of the cord blood samples (mean, 122.0 $\pm 6.6 \mathrm{fL}$; range, 114.0-137.0 fL). The mean level \pm standard deviation (SD) was $45.5 \pm 30.5 \%$ for FVIII and $19.9 \pm 12.2 \%$ for FIX. For FVIII, the range was from $1.5 \%$ to $125.0 \%$ and the $95 \%$ reference interval was $33.2 \%$ to $57.8 \%$. For FIX, the range was from 6.0\% to $52.0 \%$ and the $95 \%$ reference

Table 1. Characteristics and normal ranges of the fetal coagulation FVIII and FIX levels between 19 and 24 gestational weeks as determined in this study

\begin{tabular}{lcc}
\hline \multicolumn{1}{c}{ Characteristic } & $\begin{array}{c}\text { FVIII: C (\%) } \\
(\mathrm{n}=26)\end{array}$ & $\begin{array}{c}\text { FIX: } \mathrm{C}(\%) \\
(\mathrm{n}=29)\end{array}$ \\
\hline Age $(\mathrm{yr})$ & $29.5 \pm 4.3$ & $29.4 \pm 4.2$ \\
Gestational age at cordocentesis $(\mathrm{wk})$ & $21.5 \pm 1.4$ & $21.4 \pm 1.5$ \\
& $(19.0-24.0)$ & $(19.0-24.0)$ \\
Mean corpuscular volume $\left(\mu \mathrm{m}^{3}\right)$ & $122.1 \pm 6.8$ & $122.0 \pm 6.6$ \\
& $(114.0-137.0)$ & $(114.0-137.0)$ \\
Factor level (\%) & $45.5 \pm 30.5$ & $19.9 \pm 12.2$ \\
& $(1.5-125.0)$ & $(6.0-52.0)$ \\
95\% reference interval $(\%)$ & $33.2-57.8$ & $15.2-25.6$ \\
\hline
\end{tabular}

Values are presented as mean \pm standard deviation (range) or 95\% reference interval only.

FVIII, factor VIII; FIX, factor IX; C, coagulant activities. 
interval was $15.2 \%$ to $25.6 \%$. FVIII showed a higher SD than FIX. We examined the correlation between the levels of FVIII and FIX and the gestational age. Although the median levels of FVIII and FIX showed a decreased tendency with increasing gestational age, there was no statistical significance among gestational age groups ( $P=0.41$ for $\mathrm{FVIII}, P=0.35$ for FIX) (Table 2). Spearman correlation showed no association between gestational age and FVIII and FIX (correlation coefficient $=-0.016, P=0.951$ for FVIII; correlation coefficient $=-0.382, P=0.096$ for FIX).

Figs. $1 \mathrm{~A}$ and $\mathrm{B}$ show the normal distribution of FVIII and FIX, respectively. As the histograms suggest, the levels of both FVIII and FIX did not show normal distributions according to statistical analysis using Shapiro-Wilk test. Although FIX levels clearly showed a positively skewed distribution, FVIII levels more closely resembled a normal distribution than did the levels of FIX. If we exclude $6 \mathrm{FVIII}$ values that are equal to or greater than
$100 \%$, then the resulting distribution would be valid as a normal distribution under the Shapiro-Wilk test. Importantly, we are interested in how low the fetal coagulation factor levels can go; the lower limits show more information concerning the normal ranges greater than $95 \%$ confidence intervals. The lower limits for $\mathrm{FVIII}$ and FIX were $1.5 \%$ and $6.0 \%$, respectively.

Out of the 30 women included in the study, 18 underwent the cordocentesis procedure because they were carriers of hemophilia $A$; among these, there were two confirmed cases of the fetus (11.1\%) affected by severe hemophilia using antenatal cordocentesis. There were no cases of female carriers of hemophilia B with diagnosed fetuses. Moreover, there were no misdiagnoses in any of these cases. In addition, none of these cases had any complications, such as abortion, membrane rupture, or bleeding following diagnostic cordocentesis.

Table 2. The fetal coagulation FVIII and FIX levels according to gestational period between 19 and 24 weeks

\begin{tabular}{|c|c|c|c|c|c|}
\hline \multirow{2}{*}{ Coagulation factor } & \multicolumn{3}{|c|}{ Period (wk) } & \multirow{2}{*}{$P$-value ${ }^{a}$} & \multirow{2}{*}{$P$-value ${ }^{b}$} \\
\hline & $19-20$ & 21-22 & $23-24$ & & \\
\hline FVIII: C (\%) & $\begin{array}{c}43.0(14.0-125.0) \\
n=5\end{array}$ & $\begin{array}{c}44.5(21.0-62.0) \\
n=11\end{array}$ & $\begin{array}{c}14.0(8.0-78.0) \\
n=3\end{array}$ & 0.41 & 0.951 \\
\hline FIX: C (\%) & $\begin{array}{c}20.5(7.0-46.0) \\
n=5\end{array}$ & $\begin{array}{c}20.0(6.0-44.0) \\
n=10\end{array}$ & $\begin{array}{c}11.0(7.0-14.0) \\
n=3\end{array}$ & 0.35 & 0.096 \\
\hline
\end{tabular}

Values are presented as median (range).

${ }^{a}$ Mann-Whitney test, ${ }^{\mathrm{b}}$ Spearman correlation.

FVIII, factor VIII; FIX, factor IX.

A

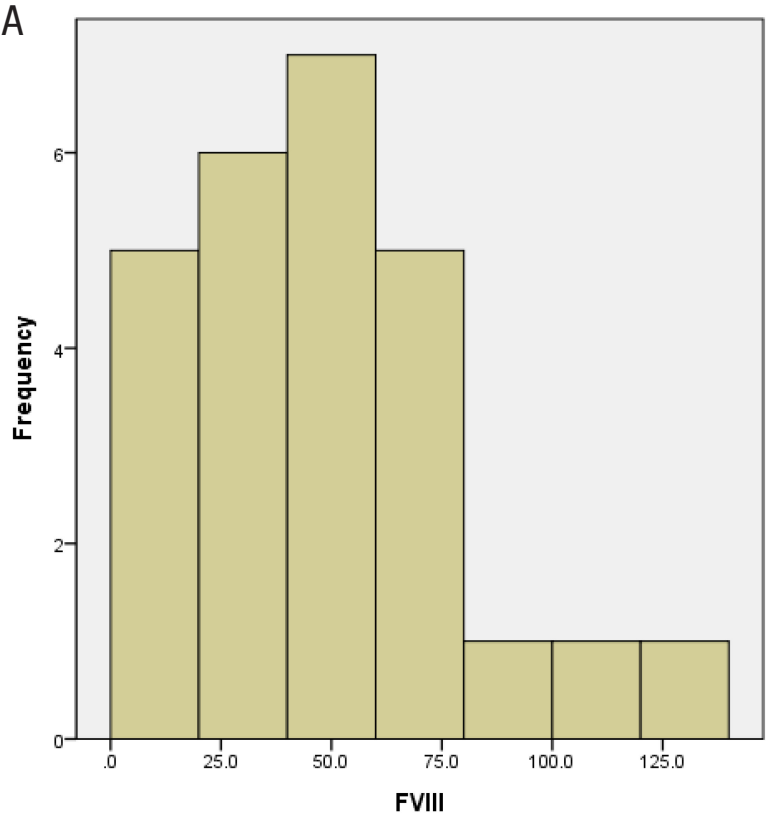

B

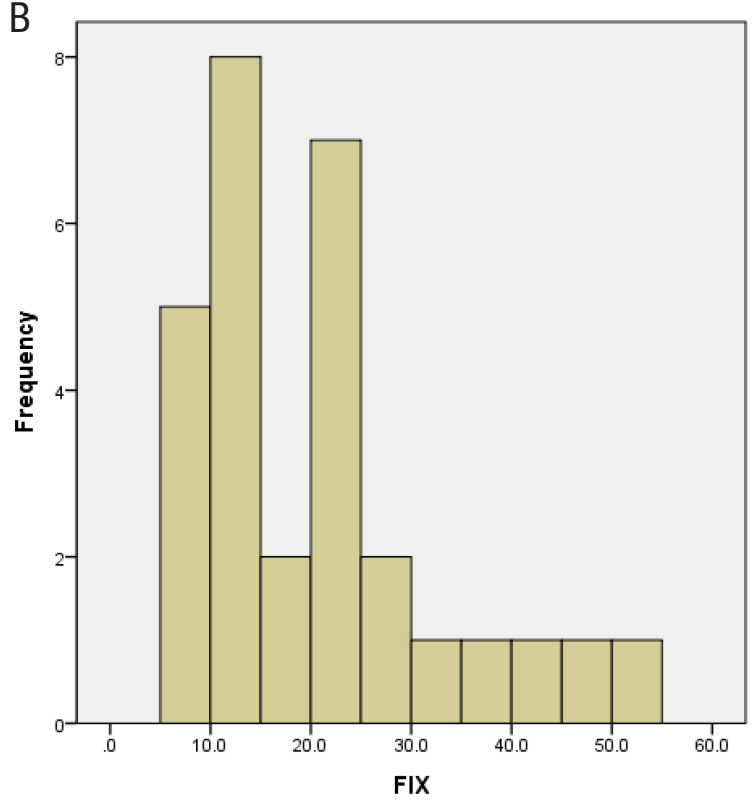

Fig. 1. (A) Normal distribution of fetal coagulation factor VIII (FVIII). (B) Normal distribution of fetal coagulation factor IX (FIX). 


\section{Discussion}

Most hemophilia cases show an inherited pattern; however, $25 \%$ of cases show a sporadic pattern originating from de novo mutations without having any affected members in the family [10]. For the inherited pattern, a son born to a mother who is a carrier would have a 50\% chance of inheriting the disease, whereas a daughter born to a mother who is a carrier would have a 50\% chance of becoming a carrier herself. All daughters born to an affected father would be obligate carriers. Before the development of adequate prenatal diagnostic techniques for hemophilia, a carrier mother commonly elected to terminate the pregnancy if the fetus was determined to be male. This, however, meant a 50\% loss of normal males; thus, effective methods for predicting fetal inheritance of the disease were needed.

Molecular genetic approaches have become common in the prenatal diagnosis of hemophilia; however, linkage analysis using polymorphism and family history is not always informative, and direct mutation detection is not yet an easily accessible approach for most people. In 2006, Shetty et al. [11] reported that a combined approach using both direct and indirect methods, including inversion of intron 22 and 1 , multiplex polymerase chain reaction (PCR), and the restriction fragment length polymorphism analysis, gave informative results in 92.2\% of patients with hemophilia A and $83.9 \%$ of patients with hemophilia B. For the remaining patients in whom molecular genetic diagnosis was uninformative, factor assay of fetal cord blood, the phenotypic approach, is the best remaining option $[5,6]$.

Determining the normal ranges for fetal coagulation factor levels is important because those levels are different from the levels of neonatal coagulation factors [12]. Several studies have attempted to determine the normal fetal ranges (Table 3) [57]. In the study of Forestier et al. in 1988 [7], the mean level of coagulation factors increased with increasing gestational age. In 2007, Shetty et al. [5] had similar result based on the gestation period. FVIII levels were more elevated and had a higher SD than FIX in all of the studies. However, none of these studies have examined Korean populations. Here, we obtained the normal ranges for a Korean population using 26 cases for FVIII and 29 cases for FIX. In our study, the normal median levels of FVIII and FIX showed decreased tendency in accordance with the gestation period even if there was no significant correlation in Mann-Whitney $U$ tests $(P=0.41$ for FVIII, $P=0.35$ for FIX) and Spearman correlation (correlation coefficient $=-0.016, P=0.951$ for FVIII; correlation coefficient $=-0.382, P=0.096$ for FIX) (Table 2). This result was different from that of another study that reported that the normal range increased in accordance with the gestation period [5,7]. We think that this may have been caused by maternal blood contamination. To avoid contamination with maternal blood and amniotic fluid, fetal blood sampling was conducted in the floating cord if possible and MCV analysis was checked at the time of cordocentesis. However, we did not use other methods to verify the purity of the fetal blood samples.

One major drawback of the fetal factor assay is the risk of fetal loss. According to a study done by Ghidini et al. [13] (1993), cordocentesis accompanies a fetal loss rate of 1.4\% if performed prior to 28 weeks of gestation. Another large cohort study involving 1,020 pregnancies was conducted by Tongsong et al. [14] in Thailand in the period from 1989 to 1999. They performed cordocentesis at mid-trimester, and the researchers compared the fetal loss rate of this group with a matched control group. Their results showed that the cordocentesis and control groups had fetal loss rates of 1.8\% and $0.7 \%$, respectively, if cordocentesis was performed before 28 weeks of gestation. However, if cordocentesis was performed after 28 weeks of gestation, the cordocentesis and control groups had fetal loss rates of $1.5 \%$ and $1.1 \%$, respectively [14].

Table 3. Normal ranges of fetal coagulation factor levels reported in the literature

\begin{tabular}{ccccc}
\hline Literature & Gestational age $(\mathrm{wk})$ & Fetuses $(\mathrm{n})$ & FVIII: C (\%) & FIX: C (\%) $^{*}$ \\
\hline Mibashan et al. (1979) [6] & $16-22$ & $22(\mathrm{FVIII})$ & $50 \pm 12.8$ & $12.5 \pm 2.4$ \\
& & $20(\mathrm{FIX})$ & & $9 \pm 2.0$ \\
Forestier et al. (1988) [7] & $18-22$ & 63 & $39 \pm 12.0$ & $10 \pm 2.0$ \\
& $23-27$ & & $41 \pm 11.0$ & $6.2 \pm 4.3$ \\
Shetty et al. (2007) [5] & $18-19$ & 28 & $23 \pm 7.2$ & $7.2 \pm 3.4$ \\
& $19-20$ & 31 & $24 \pm 10.2$ & $8.4 \pm 2.9$ \\
\hline
\end{tabular}

*Values are presented as mean \pm standard deviation.

FVIII, factor VIII; FIX, factor IX; C, coagulant activities. 
In summary, cordocentesis results in a higher rate of fetal loss than amniocentesis used for molecular genetic diagnosis. In addition, amniocentesis which was a necessary procedure for cordocentesis has a fetal loss rate of $0.5 \%$ to $1.0 \%$ [15]. Therefore, proper prenatal counseling of the mother (and family) is necessary when cordocentesis is required. The benefits and risks of the procedure should be evaluated cautiously. In fact, even though the fetal loss rate might be lower, amniocentesis and chorionic villous sampling used for molecular genetic diagnosis are also invasive procedures. Thus, both genetic and phenotypic analysis should involve proper risk stratification as long as they involve these invasive techniques $[5,16]$.

A future study involving the follow-up of the fetuses that were evaluated in this research may be beneficial. Because the normal ranges of the factor levels for the fetuses without disease are very wide, it would be interesting to determine if the differences in the fetal factor levels have any effect on the factor level during the neonatal period and in the child or adult.

Numerous studies are being performed on both polymorphisms and mutations. A recent example would be a study conducted by Park et al. [17] in 2012, in which they analyzed if Mspl and Xbal polymorphisms in intron 22 of the FVIII gene are useful in the diagnosis of Korean patients. They concluded that the linkage analysis involving those polymorphisms would be useful for carrier detection and prenatal diagnosis of hemophilia A in Korea [17]. This kind of research on polymorphisms and mutations should continue to be done in order to minimize blind spots in diagnosis where current methods are ineffective.

Microarray genetic screening is under development. Although it still requires years of optimization to be used in clinical settings, it has the potential for widespread use in detecting genetic disorders. One important advantage that it holds over DNA-based approaches is that it can also detect epigenetic changes [5]. Significant advancement in this field is expected in coming years.

Another interesting method in development is cell-free fetal DNA (cffDNA) analysis using maternal plasma. In 2006, it was reported that the analysis of cffDNA in maternal plasma rivals that of the ultrasound approach in determining fetal gender in the first trimester [18]. In 2011, Tsui et al. [19] reported that digital PCR analysis of cffDNA can provide a new non-invasive approach for genetic analysis in the prenatal diagnosis of hemophilia. Although this newly developed technique is exciting and may provide a valuable alternative to current invasive approaches, further research is necessary to better determine its advantages, disadvantages, and possible implications in clinical settings.

Our study had several limitations. First, there were only 30 cases included in this study. Second, the ranges for both of the coagulation factors were very wide, and the SD was also high.

In conclusion, we determined the normal ranges and the lowest levels of FVIII and FIX in non-affected fetuses by cord blood sampling during the mid-trimester in a Korean population. More studies are needed to better clarify the activity of these various coagulation factors and to provide accurate prenatal diagnosis of hemophilia in Korean populations; our study will provide important baseline data that will be useful in future studies.

\section{References}

1. Dahlbäck B. Blood coagulation. Lancet 2000;355:1627-32.

2. Hwang SH, Kim MJ, Lim JA, Kim HC, Kim HS. Profiling of factor VIII mutations in Korean haemophilia A. Haemophilia 2009;15:1311-7.

3. White GC 2nd, Rosendaal F, Aledort LM, Lusher JM, Rothschild C, Ingerslev J; Factor VIII and Factor IX Subcommittee. Definitions in hemophilia. Recommendation of the scientific subcommittee on factor VIII and factor IX of the scientific and standardization committee of the International Society on Thrombosis and Haemostasis. Thromb Haemost 2001;85:560.

4. Stonebraker JS, Bolton-Maggs PH, Soucie JM, Walker I, Brooker M. A study of variations in the reported haemophilia A prevalence around the world. Haemophilia 2010;16:20-32.

5. Shetty S, Ghosh K. Robustness of factor assays following cordocentesis in the prenatal diagnosis of haemophilia and other bleeding disorders. Haemophilia 2007;13:172-7.

6. Mibashan RS, Rodeck CH, Thumpston JK, Edwards RJ, Singer JD, White JM, et al. Plasma assay of fetal factors VIIIC and IX for prenatal diagnosis of haemophilia. Lancet 1979;1:1309-11.

7. Forestier F, Cox WL, Daffos F, Rainaut M. The assessment of fetal blood samples. Am J Obstet Gynecol 1988;158:1184-8.

8. Bain BJ, Bates I, Laffan MA. Dacie and Lewis practical haematology. 12th ed. London: Elsevier Health Sciences Press, 2016.

9. Over J. Methodology of the one-stage assay of Factor VIII (VIII:C). Scand J Haematol Suppl 1984;41:13-24.

10. Ghosh K, Shetty S, Tulsiani M. Evolution of prenatal diagnostic techniques from phenotypic diagnosis to gene arrays: its likely impact on prenatal diagnosis of hemophilia. Clin Appl Thromb Hemost 2009;15:277-82.

11. Shetty $S$, Ghosh K, Jijina F. First-trimester prenatal diagnosis in haemophilia A and $B$ families--10 years experience from a centre in India. Prenat Diagn 2006;26:1015-7. 
12. Holmberg $L$, Henriksson $P_{1}$ Ekelund $H_{1}$ Astedt $B$. Coagulation in the human fetus. Comparison with term newborn infants. J Pediatr 1974;85:860-4.

13. Ghidini A, Sepulveda W, Lockwood CJ, Romero R. Complications of fetal blood sampling. Am J Obstet Gynecol 1993;168:1339-44.

14. Tongsong T, Wanapirak C, Kunavikatikul C, Sirirchotiyakul S, Piyamongkol W, Chanprapaph P. Fetal loss rate associated with cordocentesis at midgestation. Am J Obstet Gynecol 2001;184:71923.

15. American College of Obstetricians and Gynecologists. ACOG practice bulletin No. 88, December 2007. Invasive prenatal testing for aneuploidy. Obstet Gynecol 2007;110:1459-67.

16. Vidal F, Farssac E, Altisent C, Puig L, Gallardo D. Rapid hemophilia
A molecular diagnosis by a simple DNA sequencing procedure: identification of 14 novel mutations. Thromb Haemost 2001;85:5803.

17. Park SH, Chung N, Lee MR, Yoo SK, Choi YM. Combined analysis of the Mspl and Xbal polymorphisms in intron 22 of the factor VIII gene for detection of hemophilia A in a Korean population. Genet Mol Res 2012;11:1-9.

18. Chi $C$, Hyett JA, Finning KM, Lee CA, Kadir RA. Non-invasive first trimester determination of fetal gender: a new approach for prenatal diagnosis of haemophilia. BJOG 2006;113:239-42.

19. Tsui NB, Kadir RA, Chan KC, Chi C, Mellars G, Tuddenham EG, et al. Noninvasive prenatal diagnosis of hemophilia by microfluidics digital PCR analysis of maternal plasma DNA. Blood 2011;117:3684-91. 